\title{
The Composition operator induced by a polynomial of degree $n$
}

\author{
Azhar Abbas Majeed* \\ Received 5, April, 2009 \\ Accepted 5, December, 2009
}

\begin{abstract}
:
In this paper, we characterize normal composition operators induced by holomorphic self-map $\varphi(z)=a z^{2}+b z+c$, when $|a| \leq 1,|b| \leq 1,|c|<1$ and $|a| \leq 1,|b| \leq 1,|c|<1$.Moreover, we study other related classes of operators, and then we generalize these results to polynomials of degree $n$.
\end{abstract}

\section{Key words: Composition operator: Normality : Unitary operator}

\section{Introduction:}

Let $\mathrm{U}$ denote the unit ball in the complex plane, the Hardy space $\mathrm{H}^{2}$ is the collection of holomorphic (analytic)functions $f(z)=\sum_{n=o}^{\infty} \hat{f}(n) z^{n}$ with $\hat{f}(n)$ denoting the $\mathrm{n}$-th Taylor coefficient of $f$ such that $\sum_{n=o}^{\infty}|\hat{f}(n)|^{2}<\infty$

and the norm is defined by $/ / f / /^{2}=\sum_{n=o}^{\infty}|\hat{f}(n)|^{2}$.

The particular importance of $\mathrm{H}^{2}$ is due to the fact that it is a Hilbert space with inner product on $\mathrm{H}^{2}$ is defined by $<f, g>=\sum_{n=0}^{\infty} \hat{f}(n) \overline{\hat{g}(n)}$, where $\hat{f}(n)$ and $\hat{g}(n)$ are $n$-th Taylor coefficient of $f$ and $g$ in $\mathrm{H}^{2}$. Let $\psi$ be a holomorphic function that take the unit ball $\mathrm{U}$ into itself (which is called holomorphic self-map of U).To each holomorphic self-map $\psi$ of $\mathrm{U}$, we associate the composition operator $C_{\psi}$ defined for all $f \in H^{2}$ by $C_{\psi} f=f \circ \psi$.

In this paper, we are going to discuss some links between the function theory and the operator theory. We investigate the relationship between the properties of symbol $\varphi$ and the operator $C_{\varphi}$ where $\varphi(z)=a z^{2}+b z+c$ such that $|a| \leq 1,|b| \leq 1,|c|<1$ and $|a| \leq 1,|b| \leq 1,|c|<1$. Composition operators have been studied by many authors in different contexts. A good source of references on the properties of composition operators on $H^{2}$ can be found in [1]. We state very loosely some basic facts on composition operators on $H^{2}$.

Theorem 1: Every composition operator $C_{\psi}$ is bounded.

Theorem 2: $C_{\psi}$ is normal if and only if $\psi(z)=\lambda z,|\lambda| \leq 1$.

Theorem 3: $C_{\delta} C_{\psi}=C_{\psi \circ \delta}$.

Theorem 4: $C_{\psi}$ is an identity operator if and only if $\psi$ is the identity map.

For each $\alpha \in U$, the reproducing kernel at $\alpha$,denoting by $K_{\alpha}$ is defined by

$$
K_{\alpha}(z)=\frac{1}{1-\bar{\alpha} z} .
$$

\footnotetext{
*University of Baghdad-College of Science-Department of Mathematics
} 
It is easily seen for each $\alpha \in U$ and $f \in H^{2}, \quad f(z)=\sum_{n=0}^{\infty} \hat{f}(n) z^{n}$ that $<f, K_{\alpha}>=\sum_{n=0}^{\infty} \hat{f}(n) \alpha^{n}=f(\alpha)$,[2].

The reproducing kernels for $H^{2}$ will play an important role in the study of composition operators since the span of this family $\left\{K_{\alpha}\right\}_{\alpha \in U}$ is a dense subset in $H^{2}$. Shapiro in [1] gave the following formula for the adjiont $C_{\psi}^{*}$ of a composition operator $C_{\psi}$ induced by a holomorphic self-map $\psi$ of $\mathrm{U}$ on that family.

Theorem 5: Let $\psi$ be a holomorphic self-map of U, then for each $\alpha \in U$ $C_{\psi}^{*} K_{\alpha}=K_{\psi(\alpha)}$.

Cowen in [3] gave an exact value of composition operator induced by polynomial of degree 1 .

Theorem 6: Let $\psi(z)=s z+t$ where $\quad \mid s / \leq 1, / t /<1 \quad$ and $|s /+| t \mid \leq 1$, the norm of $C_{\psi}$ on $H^{2}$ is defined as follows $\left\|C_{\psi} /\right\|^{2}=\frac{2}{1-|s|^{2}-|t|^{2}+\sqrt{\left(1-|s|^{2}+|t|^{2}\right)^{2}-\left.4 t\right|^{2}}}$.

In this paper, we study the normality of a composition operator induced by a holomorphic self-map $\varphi(z)=a z^{2}+b z+c \quad$ where $|a| \leq 1,|b| \leq 1,|c|<1$ and $|a| \leq 1,|b| \leq 1,|c| \leq 1$. In addition we study other related classes, and extend these results to a polynomial of degree $n$.

\section{The characterization of the normality of $C_{\varphi}$.}

Recall that [4] an operator $\mathrm{T}$ on a Hilbert space $\mathrm{H}$ is said to be normal if $T T^{*}=T^{*} T$ (where $T^{*}$ is the adjoint of $\mathrm{T})$ and is isometric if $T^{*} T=I$ (where $\mathrm{I}$ is the identity operator).
Moreover, $\mathrm{T}$ is unitary if $T T^{*}=T^{*} T=I$. We start this section by the following consequence.

\section{Theorem 1.1:}

Let $\varphi(z)=a z^{2}+b z+c, \quad$ where $|a| \leq 1,|b| \leq 1,|c|<1$ and $|a| \leq 1,|b| \leq 1,|c| \leq 1$. If $|b|=1$, then $C_{\varphi}$ is an isometric on $H^{2}$.

\section{Proof :}

Assume that $|b|=1$, so it is clear that (since $|a| \leq 1,|b| \leq 1,|c| \leq 1$ ). Therefore $\varphi(z)=b z$.To prove that $C_{\varphi}$ is isometric it is enough to show that $C_{\varphi}^{*} C_{\varphi}=I$. Since the span of the family $\left\{K_{\alpha}\right\}_{\alpha \in U}$ is dense in $H^{2}$, then we can prove the equality on this family. Let $\alpha \in U$, then by theorem (5)

$C_{\varphi}^{*} C_{\varphi} K_{\alpha}(z)=C_{\varphi}^{*} K_{\alpha}(\varphi(z))$

$=K_{\varphi(\alpha)}(\varphi(z)) \quad$ (by theorem 5)

$=\frac{1}{1-\overline{\varphi(\alpha)}(\varphi(z))}$

$=\frac{1}{1-\bar{b} \bar{\alpha} b z}$

$=\frac{1}{1-|b|^{2} \bar{\alpha} z}$

$=\frac{1}{1-\bar{\alpha} z}$

$=K_{\alpha}(z)$.

Hence $C_{\varphi}^{*} C_{\varphi} K_{\alpha}(z)=K_{\alpha}(z)$ for each $\alpha \in U$.This implies that $C_{\varphi}^{*} C_{\varphi}=I$. So $C_{\varphi}$ is isometric.

By using similar technique of (1.1) we can generalize this theorem to a polynomial of degree $n$. 


\section{Theorem 1.2:}

Let $\varphi_{n}(z)=a_{n} z^{n}+a_{n-1} z^{n-1}+\ldots+a_{1} z+a_{0}$ where $\left|a_{i}\right| \leq 1, \quad \mathrm{i}=1,2, \ldots, \mathrm{n},\left|a_{0}\right|<1$ and $\sum_{i=0}^{n}\left|a_{i}\right| \leq 1$. If $\left|a_{1}\right|=1$, then $C_{\varphi_{n}}$ is isometric operator on $H^{2}$.

The following result gives the necessary and sufficient condition for normality of $C_{\varphi}$.

Theorem 1.3:

Let $\varphi(z)=a z^{2}+b z+c$ where and $|a| \leq 1,|b| \leq 1,|c|<1$,

$|a| \leq 1,|b| \leq 1,|c| \leq 1 \quad$.Then $\quad C_{\varphi}$ is normal if and only if $\mathrm{a}=\mathrm{c}=0$.

\section{Proof :}

Assume that $C_{\varphi}$ is normal. Trivial case when $C_{\varphi}$ is the identity operator, then by theorem (4) $\varphi$ is the identity self-map, hence $\varphi(z)=z$, thus $\mathrm{a}=\mathrm{c}=0$. Therefore we may assume that $C_{\varphi}$ is not the identity operator, then $\varphi$ is not identity self-map of $U$. To prove $\mathrm{a}=\mathrm{c}=0$, we suppose the converse, first assume that $c \neq 0$ then $\varphi(0)=c \neq 0$. But $C_{\varphi}$ is normal, then $C_{\varphi} C_{\varphi}^{*} K_{0}(z)=C_{\varphi}^{*} C_{\varphi} K_{0}(z)$

[Since $C_{\varphi} K_{0}=K_{0}$ and by theorem (5) $\left.C_{\varphi}^{*} K_{0}(z)=K_{\varphi(0)}(z)\right]$

$$
C_{\varphi} K_{\varphi(0)}(z)=C_{\varphi}^{*} K_{0}(z)
$$

Hence $K_{\varphi(0)}(\varphi(z))=K_{\varphi(0)}(z)$. This implies that $\frac{1}{1-\overline{\varphi(0)} \varphi(z)}=\frac{1}{1-\overline{\varphi(0) z}}$. Thus $\overline{\varphi(0)} \varphi(z)=\overline{\varphi(0)} z$. Since $\varphi(0) \neq 0$, then $\varphi(z)=z$, which is a contradiction (since $\varphi$ is not identity map).Thus $\mathrm{c}=0$, it follows that $\varphi(z)=a z^{2}+b z$. Therefore $\varphi(0)=0$. This implies that $z^{n} H^{2}$ is an invariant subspace of $H^{2}$ under $C_{\varphi}$ for each positive integer $\mathrm{n}$ (by [5]). But $C_{\varphi}$ is normal, then by [6] $\left(z^{n} H^{2}\right)^{\perp}$ is also invariant under $C_{\varphi}$.In particular, $\left(z^{2} H^{2}\right)^{\perp}$ is an invariant subspace of $H^{2} \quad$ under $\quad C_{\varphi}$. But $\left(z^{2} H^{2}\right)^{\perp}=\operatorname{span}\{1, z\}, \quad$ then $C_{\varphi} z \in \operatorname{span}\{1, z\} \quad$.It follows that $C_{\varphi} z=\varphi(z)=\alpha+\beta z \quad$ for some $\alpha, \beta$.But $\varphi(z)=a z^{2}+b z$,therefore $\alpha=0, a=0$ and $\beta=b$,as desired.

Conversely, if $\mathrm{a}=\mathrm{c}=0$, then $\varphi(z)=b z$ with $|a| \leq 1$, then again by theorem (2) $C_{\varphi}$ is normal.

The next consequence is a generalization of (1.3).

\section{Corollary 1.4:}

Let $\varphi_{n}(z)=a_{n} z^{n}+a_{n-1} z^{n-1}+\ldots+a_{1} z+a_{0}$ where $\left|a_{0}\right|<1, \quad\left|a_{i}\right| \leq 1 \quad, \mathrm{i}=1,2, \ldots, \mathrm{n}$ and $\sum_{i=0}^{n}\left|a_{i}\right| \leq 1$.Then $C_{\varphi_{n}}$ is normal if and only if $a_{i}=0, \mathrm{i}=0, \ldots, \mathrm{n}, i \neq 1$.

\section{Corollary 1.5:}

$\mathrm{C}_{\varphi}$ is a unitary operator on $\mathrm{H}^{2}$ if and only if $/ \mathrm{b} /=1$.

\section{Proof :}

Assume that $C_{\varphi}$ is unitary, then $T T^{*}=T^{*} T=I$.

Since $C_{\varphi}$ is unitary then it is normal, thus by theorem (1.3) $\mathrm{a}=\mathrm{c}=0$, it follows that $\varphi(z)=b z,|b| \leq 1$. To show that $|b|=1$. Let $\alpha \in U, \alpha \neq 0$, then

$$
\begin{aligned}
C_{\varphi}^{*} C_{\varphi} K_{\alpha}(z) & =C_{\varphi}^{*} K_{\alpha}(\varphi(z)) \\
& =K_{\varphi(\alpha)}(\varphi(z)) \\
& =\frac{1}{1-\overline{\varphi(\alpha)} \varphi(z)} .
\end{aligned}
$$

But

$$
C_{\varphi}^{*} C_{\varphi} K_{\alpha}(z)=I\left(K_{\alpha}(z)\right)=K_{\alpha}(z) \text {. }
$$


Thus

$\frac{1}{1-\overline{\varphi(\alpha)} \varphi(z)}=\frac{1}{1-\bar{\alpha} z}$. Therefore,

$\frac{1}{1-\bar{b} \bar{\alpha} b z}=\frac{1}{1-\bar{\alpha} z}$.Hence

$\frac{1}{1-|b|^{2} \alpha z}=\frac{1}{1-\overline{\alpha z}}$. This equation satisfies

only if $|b|=1$.

Conversely, suppose that $|b|=1$. But $|a| \leq 1,|b| \leq 1,|c| \leq 1$, this follows that $\mathrm{a}=\mathrm{c}=0$. Hence by (1.3) we obtain $C_{\varphi}$ is normal, that is $C_{\varphi} C_{\varphi}^{*}=C_{\varphi}^{*} C_{\varphi}$.On the other hand, since $|b|=1$, by (1.1) $C_{\varphi}$ is isometric.

Thus $C_{\varphi} C_{\varphi}^{*}=C_{\varphi}^{*} C_{\varphi}=I$.Hence $C_{\varphi}$ is a unitary operator on $H^{2}$.

The following result can get directly from generalize (1.5).

\section{Corollary 1.6:}

Let

$\varphi_{n}(z)=a_{n} z^{n}+a_{n-1} z^{n-1}+\ldots+a_{1} z+a_{0}$ where $\left|a_{0}\right|<1, \quad\left|a_{i}\right| \leq 1, \mathrm{i}=1,2, \ldots, \mathrm{n}$ and $\sum_{i=0}^{n}\left|a_{i}\right| \leq 1$.Then $C_{\varphi_{n}}$ is a unitary operator if and only if $\left|a_{1}\right|=1$.

\section{References:}

1.Shapiro, J.H., 1993: Composition operators and classical function theory, Springer-Verlage, New York.

2.Gajath Gunatillake,2005:Spectrum of a compact weighted composition operator, phD. thesis, Purdue Uineversity.

3. Bourdon P.S., Fry E.E., Hammond C. and Spofford 2003: Norm of linear-fractional composition operators, Trans., Amer., Soc., 356(6): 2459-2480.

4. Berberain S. K., 1976: Introduction to Hilbert space, Sec. Ed., Chelesa publishing Com., New Yourk.

5. Cowen, C.C., and Keriete, T. L. III, 1988: Subnormality and composition operator on $\mathrm{H}^{2}$, J. Functiona Analysis, 81: 298- 319.

6.Radjavi H. and Rosenthal P.,1973: Invariant subspaces, SpringerVerlag, Berlin,Heidelberg, New Yourk.

\section{n المؤثر التركيبي المحتث من متعددة حدود من الارجة}

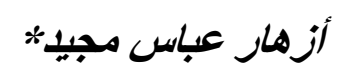

جامعة بغداد - كلية العلوم - قسم الرياضيات

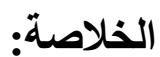

في هذا البحث أعطينا وصفاً للمؤثرات الاعتبادية المحتثة من الدالة التحليلية على U،

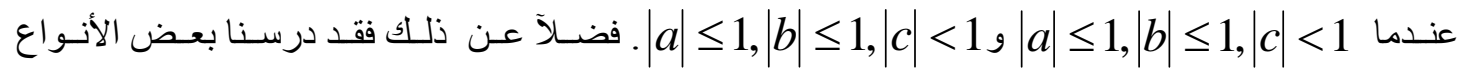
الأخرى من المؤثرات، ثم قمنا بتعميم تلأك النتائج على متعددة حدود من الدرجة النا 